\title{
Editorial
}

\section{Analysis and Models in Interdisciplinary Mathematics}

\author{
L. Jódar, ${ }^{1}$ Benito M. Chen-Charpentier, ${ }^{2}$ J.-C. Cortés, ${ }^{1}$ \\ Francisco J. Solís, ${ }^{3}$ and R.-J. Villanueva ${ }^{1}$ \\ ${ }^{1}$ Instituto Universitario de Matemática Multidisciplinar, Universitat Politècnica de València, \\ Camino de Vera $s / n, 46022$ Valencia, Spain \\ ${ }^{2}$ Department of Mathematics, University of Texas at Arlington, Arlington, TX 76019-0408, USA \\ ${ }^{3}$ CIMAT, 36000 Guanajuato, GTO, Mexico
}

Correspondence should be addressed to L. Jódar; ljodar@imm.upv.es

Received 12 March 2014; Accepted 12 March 2014; Published 19 June 2014

Copyright (C) 2014 L. Jódar et al. This is an open access article distributed under the Creative Commons Attribution License, which permits unrestricted use, distribution, and reproduction in any medium, provided the original work is properly cited.

People understand analysis concluding with numbers better than discourse analysis, because writing well is not easy and, to be exact, writing is only a benefit of genius. Analysis without numbers is always under the risk of ambiguity and lack of accuracy. Comparison with numbers is easier than with words. Mathematics is a powerful quantification tool, but we need a quantifiable model, a mathematical model.

The transit from the real problem to the mathematical model is the task of mathematical modelling. Social sciences usually waste the mathematical power of analysis, because they do not use mathematical models, just some statistics that mainly provide fixed pictures of the problem under study.

It is true that human problems are not easy because of their emotional and not always rational nature. But as humans, we are creatures of habits, we practice human herding, and we are mimetic and imitate other fellows. These characteristics make the modelling of human behaviour possible.

Mathematical innovation does not need to find new theorems or algorithms, but they may be found in the mathematical model construction that should be an acceptable representation of the real problem. Not every model is acceptable; it must be reliable in the sense that although restricted by some hypotheses, these must be clearly exposed. Important factors cannot be disregarded, and real data must be obtained.

Probably the main difference between abstract and applied analysis lies in the construction or not of a new model and the interaction with another discipline where the problem is originated. Without interaction with other disciplines or without real data, the innovation becomes empty and unreliable.

The boundary between innovation and mathematical irrelevance is subtle. Correct results could be zero innovative; just true theorems with inaccessible hypotheses are irrelevant. Thus, the innovation or novelty can arrive from the mathematical model construction or from the method to compute and simulate the model. Clear hypotheses, conclusions, and recommendations are suitable.

This special issue contains both types of contributions, those coming from the model construction and others that, starting from a given model, provide insight in the computing and simulation of the mathematical model.

This special issue contains interdisciplinary models related to the following:

(i) the propagation of opinions in microblog networks,

(ii) finding influence factors on the Spanish students selecting an engineering degree in the university,

(iii) the behaviour of consumer satisfaction,

(iv) quality supervising efficiency in logistic service providers,

(v) the risk related to threshold dividend strategies,

(vi) the identification of several formations in a set of moving entities with outliers,

(vii) forecasting Latin America's country risk scores, 
(viii) the propagation of democratic support of extreme ideologies in Spain,

(ix) oil-spill detection with swarm drones,

(x) forecasting $\mathrm{SO}_{2}$ pollution incidents,

(xi) propagation of the consumption of low invasive plastic surgery in Spain,

(xii) the effect of diffusion in a predator-prey system when predator species suffers a transmissible disease.

Methods for solving interdisciplinary models include the following:

(i) random and deterministic analytic-numerical methods for heat problems,

(ii) comparison of random methods for random ordinary differential equations,

(iii) vector algebraic algorithms to determine satellite orbits,

(iv) solving of hydrodynamic dispersion equation,

(v) error analysis for hydraulic models in water supply networks,

(vi) epidemic random network simulation in a distributed computing environment,

(vii) generalized eigenvalue problem in the neutron diffusion equation,

(viii) integration of elliptic orbital motion,

(ix) two species competitive systems with multiple delays and impulses,

(x) consistent completion of reciprocal comparison matrices,

(xi) global stability of multigroup SIRS epidemic models with varying population sizes and stochastic perturbation around equilibrium,

(xii) evaluation of supplier greenness.

\title{
Acknowledgment
}

We acknowledge all the contributors for this special issue for their participation.

\author{
L. Jódar \\ Benito M. Chen-Charpentier \\ J.-C. Cortés \\ Francisco J. Solís \\ R.-J. Villanueva
}




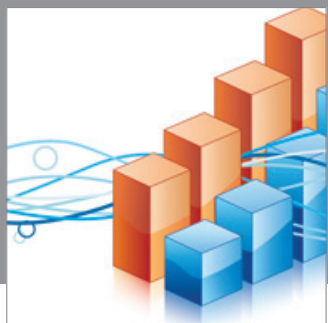

Advances in

Operations Research

mansans

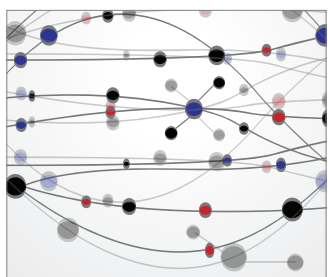

The Scientific World Journal
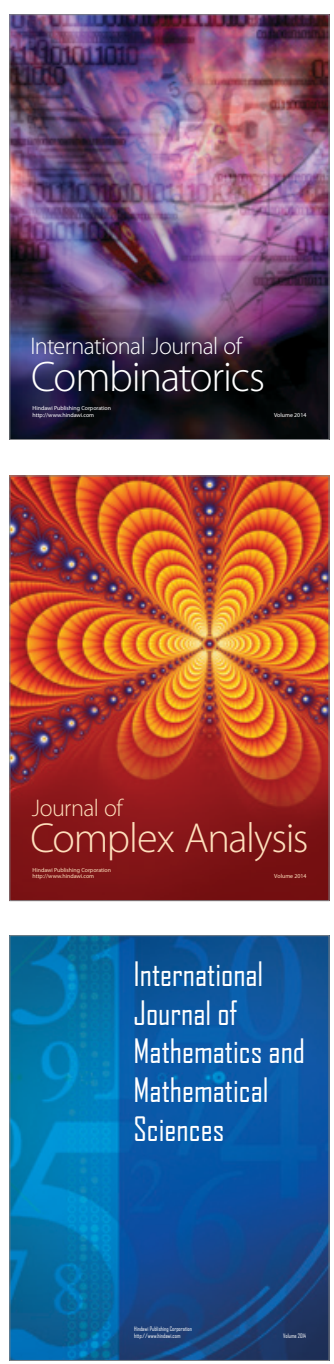
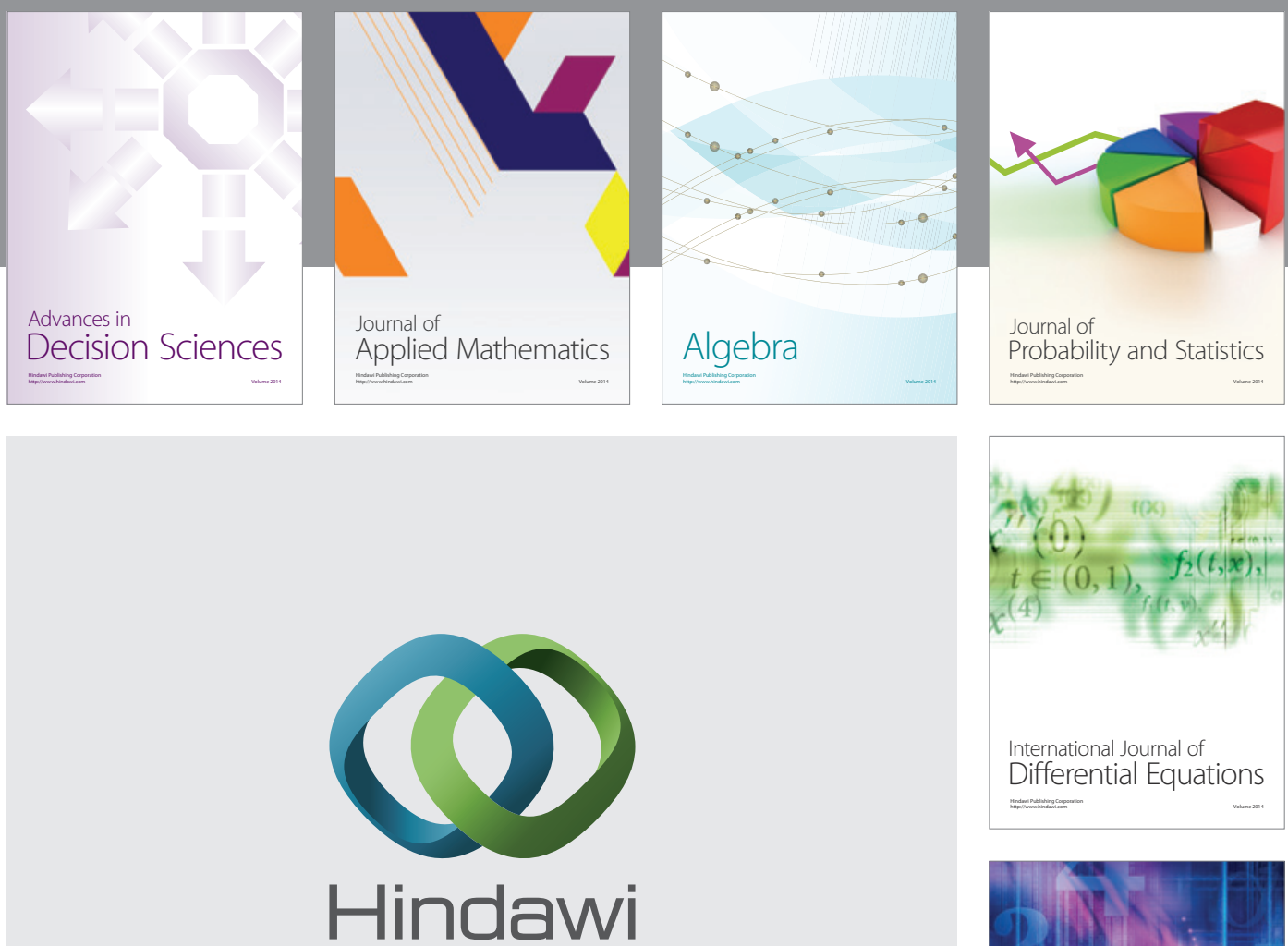

Submit your manuscripts at http://www.hindawi.com
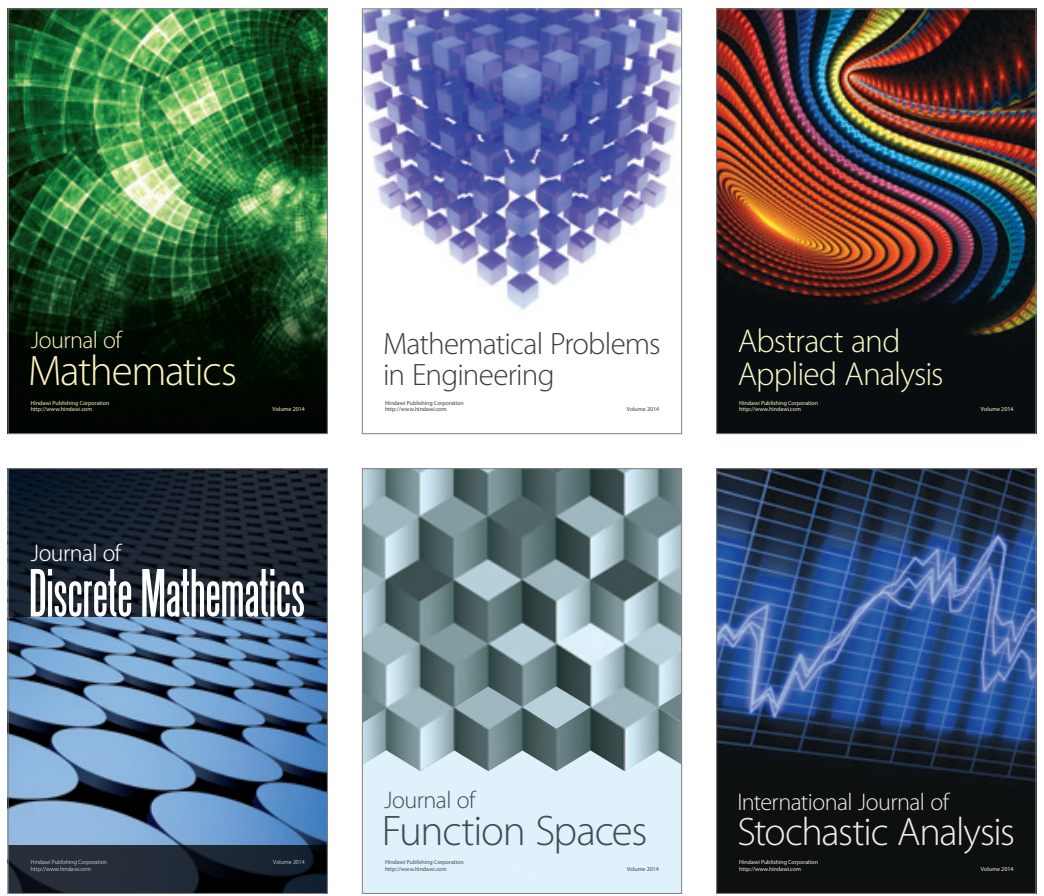

Journal of

Function Spaces

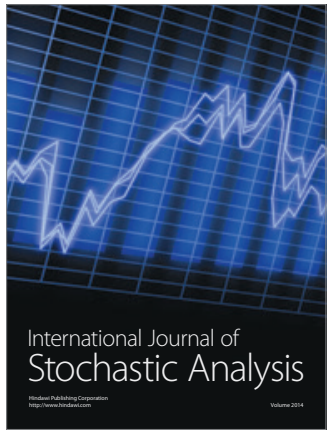

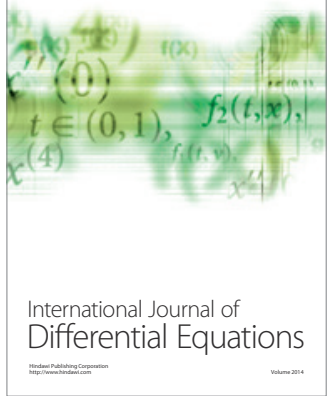
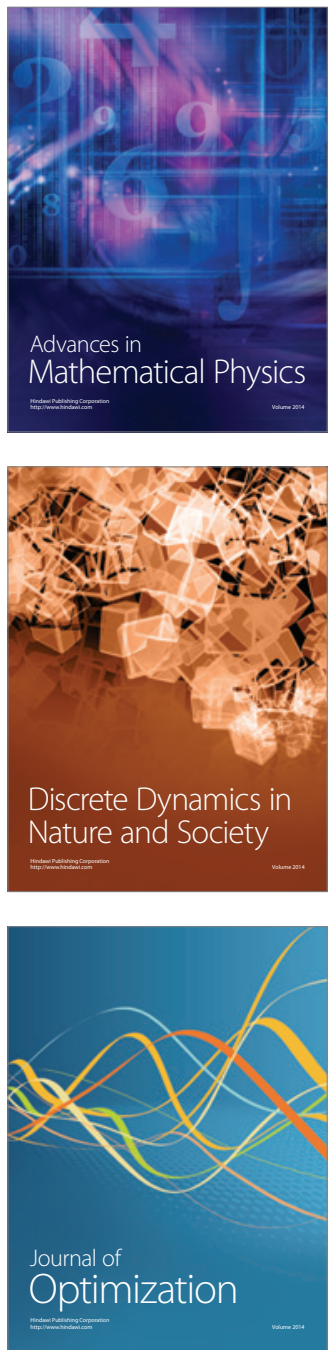